\title{
Curved spacetime theory of inhomogeneous Weyl materials
}

\author{
Long Liang ${ }^{1,2}$ and Teemu Ojanen ${ }^{2}$ \\ ${ }^{1}$ Department of Applied Physics, Aalto University School of Science, FI-00076 Aalto, Finland \\ ${ }^{2}$ Computational Physics Laboratory, Physics Unit, Faculty of Engineering and Natural Sciences, \\ Tampere University, P.O. Box 692, FI-33014 Tampere, Finland
}

(Received 19 June 2019; published 16 October 2019)

\begin{abstract}
We show how the universal low-energy properties of Weyl semimetals with spatially varying time-reversal (TR) or inversion (I) symmetry breaking are described in terms of chiral fermions experiencing curvedspacetime geometry and synthetic gauge fields. By employing Clifford representations and Schrieffer-Wolff transformations, we present a systematic derivation of an effective curved-space Weyl theory with rich geometric and gauge structure. To illustrate the utility of the formalism, we give a concrete prescription of how to fabricate nontrivial curved spacetimes and event horizons in topological insulators with magnetic textures. Our theory can also account for strain-induced effects, providing a powerful unified framework for studying and designing inhomogeneous Weyl materials.
\end{abstract}

DOI: 10.1103/PhysRevResearch.1.032006

Introduction. Semimetals and quantum liquids with linear dispersion near degeneracy points exhibit emergent relativistic physics at low energies. Topological Dirac and Weyl semimetals [1-19] have proven to be particularly fertile condensed-matter playgrounds to study the interaction of chiral fermions with gauge fields. These systems display the rich physics of quantum anomalies discovered originally in the relativistic setting [20-22]. In translationally invariant systems, the twofold band touching in Weyl semimetals can be associated with a conserved Berry charge which is topologically protected. Moreover, even in the presence of spatially varying perturbations, the low-energy properties can be understood in terms of Weyl particles experiencing artificial gravity and gauge fields [23]. The condensed-matter setting allows for remarkable opportunities in engineering synthetic gauge fields and geometries that mimic and generalize the phenomenology of high-energy physics [16,24-34].

A popular starting point for geometry and gauge-field engineering in semimetals is a strain-distorted tight-binding model [35-41]. This description, case specific to a particular lattice and orbital structure, can often be regarded as a formal device to obtain a long-wavelength theory. While being a powerful method for fabricating synthetic gauge fields, strain engineering has the limitation of producing effective geometries that are small perturbations from flat space. To obtain more general three-dimensional (3d) geometries, Ref. [23] proposed a new method of fabricating spatially varying TRand I-breaking textures. Semiclassical dynamics of carriers then reflect the interplay of effective curved geometry and Berry curvature effects. The purpose of the present work is to

Published by the American Physical Society under the terms of the Creative Commons Attribution 4.0 International license. Further distribution of this work must maintain attribution to the author(s) and the published article's title, journal citation, and DOI. establish a general and fully quantum-mechanical description of inhomogeneous Weyl semimetals. By employing Clifford representations and Schrieffer-Wolff (SW) transformations [42], we systematically consider generic TR- and I-breaking patterns and provide a controlled derivation of the low-energy Weyl Hamiltonian $H_{W}=V \sigma^{0}+e_{a}^{i}\left(k_{i}-K_{W, i}-\frac{i}{2} e_{i}^{b} \partial_{j} e_{b}^{j}\right) \sigma^{a}$. Here $\sigma^{a}$ denotes the set of Pauli matrices supplemented by a unit matrix. The effective geometry is encoded in the frame fields $e_{a}^{i}$ while the effective gauge field receives contributions from the spatial variation of the Weyl point $K_{W, i}$ and the frame fields. Our theory can also account for strain-induced effects and provides a unified low-energy description of inhomogeneous Weyl semimetals.

The obtained low-energy theory has a number of remarkable consequences. In general, spatially varying TR- and Ibreaking textures give rise to frame fields and metric tensors that mix time and space components. In contrast to mere curved space geometries realized by strain engineering, we obtain nontrivial spacetime geometries. Also, the spectral tilt of the Weyl dispersion can be tuned by TR- and I-breaking textures. To demonstrate this effect in detail, we consider $3 \mathrm{~d}$ topological insulators with magnetic textures. Strikingly, various magnetic textures give rise to Weyl semimetal phases with a spatial interface between type I and type II regions. We show that the effective geometry near the interface emulates the Schwarzschild metric at a black-hole event horizon. Our work provides powerful tools to analyze and design the properties of inhomogeneous Weyl materials.

Inhomogeneous Weyl systems. The starting point of our theory of inhomogeneous Weyl systems is a generic four-band parent state with both time-reversal and inversion symmetry intact. By introducing spatially varying TR- or I-breaking fields, the parent state is driven to an inhomogeneous Weyl semimetal phase. The minimal model for the parent states is characterized by the Hamiltonian [23]

$$
H_{0}=n(\mathbf{k}) \mathbb{I}+\kappa_{i}(\mathbf{k}) \gamma_{i}+m(\mathbf{k}) \gamma_{4},
$$


where the repeated indices are implicitly summed over, $\mathbb{I}$ is the identity matrix, and $\gamma_{\mu}$ with $\mu=1,2,3,4$ denotes the four $\gamma$ matrices satisfying anticommutation relations $\left\{\gamma_{\mu}, \gamma_{\nu}\right\}=$ $2 \delta_{\mu \nu}$. The parameters $n(\mathbf{k})$ and $m(\mathbf{k})$ are even functions of the momentum while $\kappa_{i}(\mathbf{k})$ is odd. Therefore, $\gamma_{1,2,3}$ are odd under both TR and I, while $\gamma_{4}$ is even. The fifth $\gamma$ matrix is defined as $\gamma_{5}=\gamma_{1} \gamma_{2} \gamma_{3} \gamma_{4}$, which is odd under both TR and I.

To write down the most general $4 \times 4$ Hamiltonian, we introduce ten additional matrices, $\gamma_{i j}=-i\left[\gamma_{i}, \gamma_{j}\right] / 2$. It is convenient $[13,23]$ to separate the ten matrices into three vectors $\mathbf{b}=\left(\gamma_{23}, \gamma_{31}, \gamma_{12}\right), \mathbf{b}^{\prime}=\left(\gamma_{15}, \gamma_{25}, \gamma_{35}\right)$, and $\mathbf{p}=$ $\left(\gamma_{14}, \gamma_{24}, \gamma_{34}\right)$ and one scalar $\varepsilon=\gamma_{45}$. The transformation properties of the four groups can be easily deduced from their constituent $\gamma$ matrices, and it turns out that $\mathbf{b}$ and $\mathbf{b}^{\prime}$ break TR symmetry, while $\mathbf{p}$ and $\varepsilon$ break I symmetry. The general $4 \times 4$ Hamiltonian with TR- and I-breaking terms can thus be written as

$$
H=H_{0}+\mathbf{u} \cdot \mathbf{b}+\mathbf{w} \cdot \mathbf{p}+\mathbf{u}^{\prime} \cdot \mathbf{b}^{\prime}+f \varepsilon,
$$

where the functions $\mathbf{u}, \mathbf{w}, \mathbf{u}^{\prime}$, and $f$ characterize the symmetrybreaking fields which are position dependent. Because $\mathbf{r}$ is even under TR but odd under I, w and $f$ should be even functions of $\mathbf{r}$ to break the inversion symmetry. If $\mathbf{u}$ and $\mathbf{u}^{\prime}$ are not even functions of $\mathbf{r}$, the inversion symmetry is also broken. But since $\mathbf{u}$ and $\mathbf{u}^{\prime}$ fields always break time-reversal symmetry irrespective of their $\mathbf{r}$ dependence, we label them as time-reversal symmetry-breaking terms. In general, we regard TR- and I-breaking terms as smooth functions of position $\mathbf{r}$ and assume that $\mathbf{r}$ and $\mathbf{k}$ are conjugate variables $\left[r_{i}, k_{j}\right]=$ $i \delta_{i j}$. Elastic deformations and strain typically induce spatial dependence in $H_{0}$ [35-40]. While we are mainly considering inhomogeneous TR and I breaking, we will discuss below how strain is included in our formalism in the continuum limit.

TR-breaking case. Here we derive an effective curvedspace Weyl equation for inhomogeneous TR-breaking systems in two opposite limits, vanishing mass $(m=0)$ and large mass. Physically these correspond to metallic and insulating parent states. We consider the $\mathbf{u}(\mathbf{r}) \cdot \mathbf{b}$ term which corresponds to $3 \mathrm{~d}$ magnetization or any field which transforms as magnetization under TR and spatial rotations. The unit matrix term $n(\mathbf{k})$ can be set as zero since it only shifts the energy. For the $m(\mathbf{k})=0$ case, the Hamiltonian can be readily block diagonalized. Defining two sets of Pauli matrices $\sigma_{i}$ and $\tau_{i}\left(\sigma_{0}\right.$ and $\tau_{0}$ are the $2 \times 2$ unit matrix) and working in the chiral representation $\gamma_{i}=\tau_{3} \otimes \sigma_{i}, \gamma_{4}=\tau_{1} \otimes \sigma_{0}$, the Hamiltonian splits to two blocks

$$
H_{W}^{ \pm}=\left[u_{i}(\mathbf{r}) \pm \kappa_{i}(\mathbf{k})\right] \sigma_{i}=d_{i}^{ \pm}(\mathbf{k}, \mathbf{r}) \sigma_{i} .
$$

The local Weyl point are determined by $d_{i}^{ \pm}=u_{i}(\mathbf{r}) \pm$ $\kappa_{i}\left(\mathbf{K}_{W}\right)=0$. Thus, $\mathbf{u}(\mathbf{r})$ gives rise to an axial gauge field while the frame fields can be straightforwardly obtained as indicated [by Eq. (8)] below.

The complementary regime of nonzero mass gives rise to rich geometric and gauge structure. It is convenient to parametrize the symmetry-breaking fields as $\mathbf{u}(\mathbf{r})=$ $u(\mathbf{r})[\sin \theta(\mathbf{r}) \cos \phi(\mathbf{r}), \sin \theta(\mathbf{r}) \sin \phi(\mathbf{r}), \cos \theta(\mathbf{r})]$. To derive an effective Weyl Hamiltonian, we first rotate the fields along the $z$ direction by applying a unitary transformation, $W^{\dagger}(\mathbf{r}) \mathbf{u} \cdot \mathbf{b} W(\mathbf{r})=u(\mathbf{r}) b_{3}$. This is achieved by choosing $W=\exp \left(-i \phi b_{3} / 2\right) \exp \left(-i \theta b_{2} / 2\right)$. The Hamiltonian after the transformation becomes

$$
H^{\prime}=\frac{1}{2}\left\{E_{a}^{i} \gamma^{a}, \kappa_{i}(\mathbf{k}-\omega)\right\}+m(\mathbf{k}-\omega) \gamma_{4}+u \gamma_{12} .
$$

The anticommutator structure results from the noncommutativity of position and momentum. Here $E_{a}^{i}$ with $i$ and $a$ running from 1 to 3 are frame fields defined through $W^{\dagger} \gamma_{i} W=E_{a}^{i} \gamma^{a}$. Using the frame fields, we can define a metric $g^{i j}=E_{a}^{i} E_{b}^{j} \eta^{a b}$ with $\eta$ being the Minkowski metric, $\eta=\operatorname{diag}(-1,1,1,1)$. The indices $i, j, k$ can be raised or lowered by $g^{i j}$ or its inverse $g_{i j}$, and $a, b, c$ are raised or lowered by $\eta^{a b}$ or $\eta_{a b}$. It is easy to verify that $E_{a}^{i} E_{i}^{b}=\delta_{a}^{b}$ and $E_{a}^{i} E_{j}^{a}=\delta_{j}^{i}$. The spin connection $\boldsymbol{\omega}=i W^{\dagger} \partial_{\mathbf{r}} W$ can be written in terms of the frame fields as $\omega_{i}=\omega_{i}^{a b} \gamma_{a b}=-E_{j}^{a} \nabla_{i} E^{j b} \gamma_{a b} / 4$, with $\nabla$ being the covariant derivative on the manifold. Alternatively, $\omega$ can also be viewed as a $\mathrm{SU}(2)$ gauge connection $\omega_{i}=\omega_{i}^{j} b_{j}$. Equation (4) describes a Dirac electron moving in a curved space [43] in the presence of time-reversal symmetry-breaking field $u$. The elegant gauge structure of Eq. (2) is discussed in detail in the Supplemental Material (SM) [44]. While the Dirac equation still describes flat space $g^{i j}=\delta^{i j}$, it contains redundant high-energy bands. The curved space geometry and gauge fields emerge as we project the four-band Dirac Hamiltonian, Eq. (4), to the two-band low-energy Weyl Hamiltonian. If the symmetry-breaking field $\mathbf{u}$ is constant, this can be done exactly by a momentum-dependent unitary transformation. However, for the position-dependent fields we are interested in, this method is no longer exact since the momentum and position do not commute with each other. There will be additional terms related to the derivatives of the fields that mix the high- and low-energy degrees of freedom, and in general it is impossible to perform the block diagonalization. However, in the large-mass limit and slowly varying fields, we can derive an effective low-energy theory in a controlled way by employing the SW transformation.

First, we expand Eq. (4) to the first order in derivatives of the symmetry-breaking field. This yields

$$
H^{\prime} \approx \tilde{\kappa}_{i}(\mathbf{k}, \mathbf{r}) \gamma_{i}+m(\mathbf{k}) \gamma_{4}+u \gamma_{12}+u_{i}^{\prime} b_{i}^{\prime}+f \gamma_{45},
$$

where $\quad \tilde{\kappa}_{i}=\left\{\kappa_{i}(\mathbf{k}), E_{a}^{i}(\mathbf{r})\right\} / 2, \quad u_{i}^{\prime}=\partial_{k_{j}} m \omega_{j}^{i}, \quad$ and $\quad f=$ $\partial_{k_{j}} \kappa_{i} \omega_{j}^{a} E_{a}^{i}$. Choosing a particular representation $\gamma_{1}=\tau_{0} \otimes \sigma_{x}$, $\gamma_{2}=\tau_{0} \otimes \sigma_{y}, \quad \gamma_{3}=\tau_{x} \otimes \sigma_{z}$, and $\gamma_{4}=\tau_{z} \otimes \sigma_{z}$, the terms proportional to $\gamma_{1,2,4}, b_{3}$, and $b_{3}^{\prime}$ are block diagonal. Employing the SW transformation, we seek matrix $S$ such that the unitary equivalent Hamiltonian $e^{S} H^{\prime} e^{-S}$ is block diagonal. In the SM, we explicitly write $S$ in the lowest order in the large-mass limit $u_{i}^{\prime} / m, \kappa_{3}^{\prime} / m \ll 1$. The transformed Hamiltonian is block diagonal, yielding an effective Weyl Hamiltonian

$$
H_{W}=d_{a}(\mathbf{k}, \mathbf{r}) \sigma^{a},
$$

with $\quad d_{i=1,2}=\tilde{\kappa}_{i}-f u_{i}^{\prime} /(2 m)-f u_{i}^{\prime} /(2 u), \quad d_{3}=-m+u-$ $\left(\tilde{\kappa}_{3}^{2}+f^{2}\right) /(2 m)+\left(u_{1}^{\prime 2}+u_{2}^{\prime 2}\right) /(2 u)$, and $d_{0}=u_{3}^{\prime}+f \tilde{\kappa}_{3} / m$. The Weyl points are determined by $d_{i=1,2,3}=0$, and due to the inversion symmetry, if $\mathbf{K}_{W}$ is a Weyl point, $-\mathbf{K}_{W}$ will also be a Weyl point. The higher order corrections to Eq. (6) are proportional to $\partial_{k}^{2} \kappa_{i}\left(\partial_{r} \mathbf{u}\right)^{2}, \partial_{k}^{2} m\left(\partial_{r} \mathbf{u}\right)^{2}$, which are always small for smooth $\mathbf{u}$ and vanish completely for $H_{0}$ with a linear dispersion. Expanding the Hamiltonian around $\mathbf{K}_{W}$, we arrive 
at our main result,

$$
H_{W} \approx V \sigma^{0}+e_{a}^{i}\left(k_{i}-K_{W, i}-\frac{i}{2} e_{i}^{b} \partial_{j} e_{b}^{j}\right) \sigma^{a},
$$

where $i=1,2,3$ and $a=0,1,2,3$. The Weyl point depends on the position and can be interpreted as $U(1)$ vector potential, and $V=d_{0}\left(\mathbf{K}_{W}\right)-\frac{i}{2} e_{0}^{b} \partial_{j} e_{b}^{j}$ acts as an effective scalar potential. The potentials acquire corrections from the frame fields, ensuring the Hamiltonian Hermitian. The frame fields are defined through

$$
e_{a}^{i}(\mathbf{r})=\left.\frac{\partial d_{a}}{\partial k_{i}}\right|_{\mathbf{K}_{W}},
$$

with additions $e_{0}^{0}=-1$ and $e_{1,2,3}^{0}=0$. We obtain from the frame fields the emergent metric $g^{\mu \nu}=e_{a}^{\mu} e_{b}^{\nu} \eta^{a b}$. Explicitly,

$$
g^{00}=-1, \quad g^{0 i}=e_{0}^{i}, \quad g^{i j}=-e_{0}^{i} e_{0}^{j}+e_{a}^{i} e_{a}^{j} .
$$

It should be stressed that, as indicated by the effective metric tensor with space and time mixing terms $g^{0 i}$, the emergent geometry of Eq. (7) is fundamentally different from the inhomogeneous strain-induced metrics that do not contain the mixing terms. The dispersion relation of the Weyl fermion can be determined by $g^{\mu v} p_{\mu} p_{v}=0$ with $p=(\omega, \mathbf{k})$, which gives

$$
\omega=e_{0}^{i} k_{i} \pm \sqrt{e_{l}^{i} e_{l}^{j} k_{i} k_{j}} .
$$

If $\left|e_{0}^{i} e_{i}^{l}\right|<1$, it is a type I Weyl semimetal, and if $\left|e_{0}^{i} e_{i}^{l}\right|>1$ we obtain a type II Weyl semimetal with overtilted Weyl cone. Remarkably, as shown below, this fact can be employed in engineering a spatial interface between type I and type II Weyl semimetals. The interface between type I and type II Weyl semimetals, which could simulate properties of the black hole horizon, may be designed experimentally by controlling the magnetic texture. This could open a method to study Hawking radiation [45-47] and quantum chaos [48-51] in Weyl semimetals.

To conclude this section, we outline how things would have changed had we considered the other TR-breaking triplet $\mathbf{u}^{\prime} \cdot \mathbf{b}^{\prime}$ instead of $\mathbf{u} \cdot \mathbf{b}$. In this case, we can use essentially the same method to derive a two-band model, but in general this leads to a nodal line semimetal [13] instead of a Weyl semimetal. While interesting, this case is not relevant for Weyl-type behavior and is postponed to the SM.

I-breaking case. The derivation of the curved-space Weyl Hamiltonian can be extended to I-breaking systems. Several Weyl materials that break inversion (but preserve TR) symmetry have be observed [15-17]. In general, a spatially varying I-breaking term $\mathbf{w}(\mathbf{r}) \cdot \mathbf{p}$ can be treated similarly as the TR-breaking case. This has been carried out in the SI where we obtain an I-breaking variant of Eq. (6). The essential difference from the TR-breaking case is that the role of the mass term $m$ is played by $\tilde{\kappa}_{1}$ or $\tilde{\kappa}_{2}$. Thus, the controlling parameter in this situation is $\tilde{\kappa}_{1}$ (or $\tilde{\kappa}_{2}$ ) instead of $m$. Assuming $\tilde{\kappa}_{1}$ sufficiently large, which is also a required to obtain Weyl points in this case, we can find a SW transformation to block diagonalize $H^{\prime}$ and obtain a two-band Hamiltonian. Finally, analogously to the $\mathbf{u}^{\prime} \cdot \mathbf{b}^{\prime}$ term, the $f \gamma_{45}$ term as a sole Ibreaking term leads to a nodal line semimetal as shown in the SM.
Application I: Engineering tilts and horizons. Here, we illustrate the power and utility of the developed formalism by proposing concrete systems with spatial interface between type I and type II Weyl fermions. We consider a simple parent model with $\kappa_{i}=k_{i}$ and $m(\mathbf{k})=m$ which could be realized in $3 \mathrm{~d}$ TR-invariant insulators with magnetization texture [23] or in the topological insulator-magnet heterostructures [12]. The TR-breaking field, representing, for example, $3 \mathrm{~d}$ magnetization, is parametrized as $\mathbf{u}=$ $u(\mathbf{r})(\sin \theta \cos \phi, \sin \theta \sin \phi, \cos \theta)$ with spatially varying angles.

Following the procedure discussed above, we obtain an effective $2 \times 2$ Hamiltonian with $d_{1}=k_{x} \cos \theta \cos \phi+$ $k_{y} \cos \theta \sin \phi-k_{z} \sin \theta, d_{2}=-k_{x} \sin \phi+k_{y} \cos \phi, d_{3}=u-$ $m-\left(\tilde{\kappa}_{3}^{2}+f^{2}\right) /(2 m)$, and $d_{0}=-\tilde{\kappa}_{3} f / m$, where $\tilde{\kappa}_{3}=$ $k_{x} \cos \phi \sin \theta+k_{y} \sin \phi \sin \theta+k_{z} \cos \theta \quad$ and $\quad f=\left(\partial_{r_{z}} \phi+\right.$ $\left.\cos \phi \partial_{r_{y}} \theta-\sin \phi \partial_{r_{x}} \theta\right) / 2$. The products of $\mathbf{k}$ - and $\mathbf{r}$-dependent terms should be understood as symmetrized. For the lowest-order of the SW transformation to be a good approximation around the Weyl point, the condition $u-m \ll m$ should be satisfied. The Weyl points are determined by $\pm \mathbf{K}_{W}= \pm K_{W}(\sin \theta \cos \phi, \sin \theta \sin \phi, \cos \theta)$ with $K_{W}=\sqrt{2 m(u-m)-f^{2}}$. For simplicity, we assume that $2 m(u-m)-f^{2}>0$ such that there always exist two well-separated Weyl points. Expanding around $\mathbf{K}_{W}$, we obtain the linearized Weyl Hamiltonian with the frame fields being $e_{0}^{0}=-1, \quad e_{0}^{i}=-f / m(\sin \theta \cos \phi, \sin \theta \sin \phi, \cos \theta), \quad e_{1}^{i}=$ $(\cos \theta \cos \phi, \cos \theta \sin \phi,-\sin \theta), \quad e_{2}^{i}=(-\sin \phi, \cos \phi, 0)$, and $e_{3}^{i}=-K_{W} / f e_{0}^{i}$. After straightforward calculations, we find $e_{0}^{i} e_{i}^{x}=e_{0}^{i} e_{i}^{y}=0$ and $e_{0}^{i} e_{i}^{z}=f / K_{W}$. Therefore, if $\left|f / K_{W}\right|<1$, the node is of type I, if $\left|f / K_{W}\right|>1$, it is of type II, and the interface between the type I and type II regions, i.e., the event horizon, is determined by $\left|f / K_{W}\right|=1$. In the $\mathrm{SM}$, it is shown that in a suitable local basis the metric is analogous to the Schwarzschild metric near the horizon in the Gullstrand-Painlevé coordinates [45,52,53].

Having worked out the general case, we now study a special case with $u$ as a constant, $\phi=0$, and $\theta=r / \xi$ with $r=$ $\sqrt{x^{2}+y^{2}+z^{2}}$. Correspondingly, we find $f=y /(2 r \xi)$. This magnetic texture is slowly varying in the length scale much smaller than $\xi$. Since $0 \leqslant|f| \leqslant 1 /(2 \xi)$, there always exists a type I region, and to have a type II region, the condition $4 m(u-m) \xi^{2}<1$ should be satisfied. The event horizon is determined by $y^{2}=4 \xi^{2} m(u-m) r^{2}$, which defines a conical surface; see Figs. 1(a) and 1(b). Clearly, the interface can be tuned when it is possible to manipulate $\xi$. Thus, the horizon may be tuned experimentally, providing a way to simulate the Hawking radiation using Weyl semimetals [45-47]. As depicted by Figs. 1(c) and 1(d), different shapes of the event horizon can also be realized; see the SM for more details.

Application II: Inclusion of strain effects. As noted above, there exists a significant body of literature on strain-induced artificial gauge fields and synthetic geometry [35-41]. In these treatments, the strain effects manifest at the level of the TRand I-preserving parent state Hamiltonian $H_{0}$ which becomes position dependent. In this respect, the strain engineering can be viewed as complementary to the studied case with spatially dependent TR- and I-breaking fields. However, here we show how to incorporate the strain effects to a unified theory of 
(a)

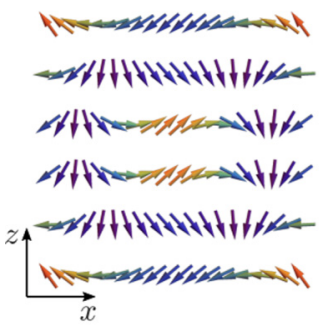

(c)

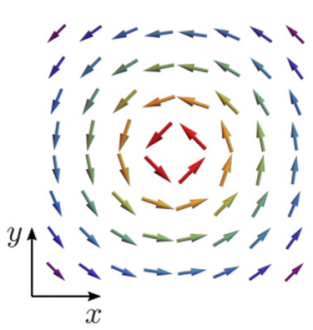

(b)

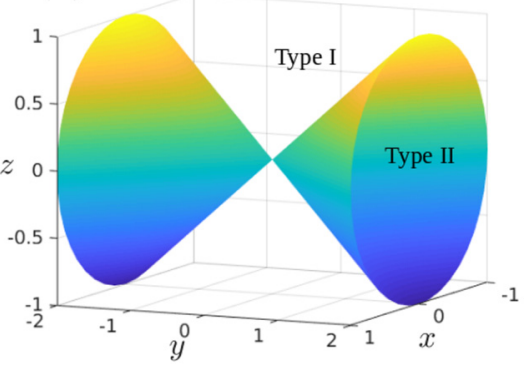

(d)

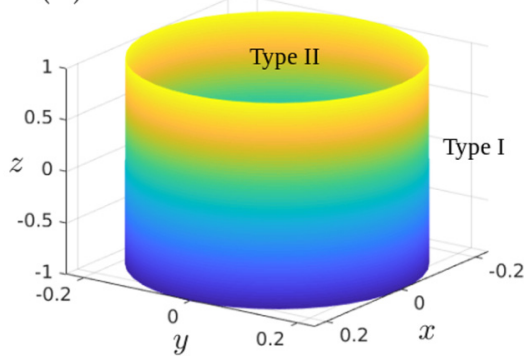

FIG. 1. Magnetic textures and the corresponding event horizons. [(a), (b)] $\mathbf{u}=u(\sin r / \xi, 0, \cos r / \xi)$ with $u / m=11 / 10$ and $m \xi=\sqrt{2}$. The horizon is a conical surface defined by $x^{2}+z^{2}=$ $y^{2} / 4$. [(c), (d)] $\mathbf{u}=\left(-u_{\|} y / \sqrt{x^{2}+y^{2}}, u_{\|} x / \sqrt{x^{2}+y^{2}}, u_{3}\right)$, where $u_{\|}=u_{0} \exp \left(-\sqrt{x^{2}+y^{2}} / \xi\right), u_{3} / m=10 / 9, u_{0} / u_{3}=1 / 9$, and $m \xi=$ $9 / 70$. The horizon is a cylinder surface with the radius about $0.232 \xi$. The values of the coordinates in both plots are given in the units of $\xi$.

inhomogeneous Weyl systems in the continuum limit. In the presence of small strain, the spatial metric is related to the strain tensor $u_{i j}$ as $g_{i j}=\delta_{i j}+2 u_{i j}$, and the frame fields can be chosen as $\bar{E}_{a}^{i}=\delta_{a}^{i}-\delta_{a j} u^{i j}$ [35]. Employing these frame fields $\bar{E}_{a}^{i}$ and the corresponding spin connection $\overline{\boldsymbol{\omega}}$, the four-band Dirac Hamiltonian in the presence of strain- and TR-breaking field $\mathbf{u}(\mathbf{r}) \cdot \mathbf{b}$ can be written in a Hermitian form as (we assume $\kappa_{i}=k_{i}$ and $\left.m(\mathbf{k})=m\right)$

$$
H_{0}=\frac{1}{2}\left\{\bar{E}_{a}^{i} \gamma^{a}, k_{i}-\bar{\omega}_{i}\right\}+m \gamma_{4}+\mathbf{u}(\mathbf{r}) \cdot \mathbf{b} .
$$

This prescription can be understood as a momentumdependent minimal substitution [26] accounting for strain. The strain-induced gauge fields $[37,54]$ are encoded in the frame fields and the spin connection [35,55]. Using the same unitary transformation $W$ as previously, we can rotate $\mathbf{u} \cdot \mathbf{b}$ along the $z$ direction, and the Hamiltonian after rotation becomes

$$
H^{\prime}=\frac{1}{2}\left\{\tilde{E}_{a}^{i} \gamma^{a}, k_{i}-\tilde{\omega}_{i}\right\}+m \gamma_{4}+u(\mathbf{r}) b_{3},
$$

where $\tilde{E}_{a}^{i}=\bar{E}_{b}^{i} E_{a}^{b}$ and $\tilde{\boldsymbol{\omega}}=W^{\dagger} \overline{\boldsymbol{\omega}} W+\boldsymbol{\omega}$ are the new frame fields and the modified spin connection containing the combined effects of strain and inhomogeneous TR breaking. In the SM, we show that the spin connection transforms exactly in agreement with the modification of the frame fields. Thus, after inclusion of strain effects, Eq. (12) takes mathematically the same form as Eq. (4) without strain. We can proceed with the projection to the low-energy space precisely as before to obtain an effective Weyl Hamiltonian of form Eq. (7), now accounting for the strain and inhomogeneous TR-breaking texture.

Summary and outlook. By employing Clifford representations and Schrieffer-Wolff transformations, we carried out a controlled derivation of quantum-mechanical low-energy theory for chiral fermions in Weyl semimetals with smooth TRand I-breaking textures. The resulting effective Weyl Hamiltonian, describing carriers experiencing an effective curved spacetime, provides a unified approach also in the presence of strain. To illustrate the utility of the developed formalism, we proposed a concrete prescription to realize a spatial type I-type II interface in magnetic topological insulators. This interface is mathematically analogous to an event horizon of a black hole and may provide an experimental access to exotic high-energy phenomena. The developed low-energy theory is applicable to a wide variety of inhomogeneous Weyl semimetals and provides a powerful framework for analyzing and designing these systems.

An interesting avenue for future work is the generalization of our theory to time-dependent TR- and I-breaking textures that give rise to nonstationary geometries and gauge fields. Another intriguing problem concerns the connections between different geometric responses in Weyl semimetals. As highlighted in the present work, low-energy carriers respond to magnetization through the change of effective geometry. This is analogous to elastic deformations in response to stress. Furthermore, thermal transport coefficients are also related to effective geometry through gravitational response $[31,56]$. These facts lead us to speculate on possible novel connections between seemingly distinct (magnetic, elastic, and thermal) response properties.

Acknowledgment. The authors acknowledge Aalto Center for Quantum Engineering for financial support.
[1] G. E. Volovik, The Universe in a Helium Droplet (Oxford University Press, Oxford, UK, 2003).

[2] S. Murakami, Phase transition between the quantum spin Hall and insulator phases in 3D: Emergence of a topological gapless phase, New J. Phys. 9, 356 (2007).

[3] S. M. Young, S. Zaheer, J. C. Y. Teo, C. L. Kane, E. J. Mele, and A. M. Rappe, Dirac Semimetal in Three Dimensions, Phys. Rev. Lett. 108, 140405 (2012).

[4] Z. Wang, Y. Sun, X.-Q. Chen, C. Franchini, G. Xu, H. Weng, $\mathrm{X}$. Dai, and Z. Fang, Dirac semimetal and topological phase transitions in $A_{3} \mathrm{Bi}(A=\mathrm{Na}, \mathrm{K}, \mathrm{Rb})$, Phys. Rev. B 85, 195320 (2012).
[5] Z. Wang, H. Weng, Q. Wu, X. Dai, and Z. Fang, Threedimensional Dirac semimetal and quantum transport in $\mathrm{Cd}_{3} \mathrm{As}_{2}$, Phys. Rev. B 88, 125427 (2013).

[6] Z. K. Liu, B. Zhou, Y. Zhang, Z. J. Wang, H. M. Weng, D. Prabhakaran, S. K. Mo, Z. X. Shen, Z. Fang, X. Dai, Z. Hussain, and Y. L. Chen, Discovery of a three-dimensional topological Dirac semimetal, $\mathrm{Na}_{3} \mathrm{Bi}$, Science 343, 864 (2014).

[7] M. Neupane, S.-Y. Xu, R. Sankar, N. Alidoust, G. Bian, C. Liu, I. Belopolski, T.-R. Chang, H.-T. Jeng, H. Lin, A. Bansil, F. Chou, and M. Z. Hasan, Observation of a three-dimensional topological Dirac semimetal phase in high-mobility $\mathrm{Cd}_{3} \mathrm{As}_{2}$, Nat. Commun. 5, 3786 (2014). 
[8] Z. K. Liu, J. Jiang, B. Zhou, Z. J. Wang, Y. Zhang, H. M. Weng, D. Prabhakaran, S.-K. Mo, H. Peng, P. Dudin et al., A stable three-dimensional topological Dirac semimetal $\mathrm{Cd}_{3} \mathrm{As}_{2}$, Nat. Mater. 13, 677 (2014).

[9] X. Wan, A. M. Turner, A. Vishwanath, and S. Y. Savrasov, Topological semimetal and Fermi-arc surface states in the electronic structure of pyrochlore iridates, Phys. Rev. B 83, 205101 (2011).

[10] S. Borisenko, Q. Gibson, D. Evtushinsky, V. Zabolotnyy, B. Büchner, and R. J. Cava, Experimental Realization of a ThreeDimensional Dirac Semimetal, Phys. Rev. Lett. 113, 027603 (2014).

[11] G. Xu, H. Weng, Z. Wang, X. Dai, and Z. Fang, Chern Semimetal and the Quantized Anomalous Hall Effect in $\mathrm{HgCr}_{2} \mathrm{Se}_{4}$, Phys. Rev. Lett. 107, 186806 (2011).

[12] A. A. Burkov and L. Balents, Weyl Semimetal in a Topological Insulator Multilayer, Phys. Rev. Lett. 107, 127205 (2011).

[13] A. A. Burkov, M. D. Hook, and L. Balents, Topological nodal semimetals, Phys. Rev. B 84, 235126 (2011).

[14] G. B. Halász and L. Balents, Time-reversal invariant realization of the Weyl semimetal phase, Phys. Rev. B 85, 035103 (2012).

[15] B. Q. Lv, H. M. Weng, B. B. Fu, X. P. Wang, H. Miao, J. Ma, P. Richard, X. C. Huang, L. X. Zhao, G. F. Chen et al., Experimental Discovery of Weyl Semimetal TaAs, Phys. Rev. X 5, 031013 (2015).

[16] X. Huang, L. Zhao, Y. Long, P. Wang, D. Chen, Z. Yang, H. Liang, M. Xue, H. Weng, Z. Fang, X. Dai, and G. Chen, Observation of the Chiral-Anomaly-Induced Negative Magnetoresistance in 3d Weyl Semimetal TaAs, Phys. Rev. X 5, 031023 (2015).

[17] S.-Y. Xu, I. Belopolski, N. Alidoust, M. Neupane, G. Bian, C. Zhang, R. Sankar, G. Chang, Z. Yuan, C.-C. Lee et al., Discovery of a Weyl fermion semimetal and topological Fermi arcs, Science 349, 613 (2015)

[18] Y. Xu, F. Zhang, and C. Zhang, Structured Weyl Points in Spin-Orbit Coupled Fermionic Superfluids, Phys. Rev. Lett. 115, 265304 (2015).

[19] A. A. Soluyanov, D. Gresch, Z. Wang, Q. Wu, M. Troyer, X. Dai, and B. A. Bernevig, Type-II Weyl semimetals, Nature (London) 527, 495 (2015).

[20] S. L. Adler, Axial-vector vertex in spinor electrodynamics, Phys. Rev. 177, 2426 (1969).

[21] J. S. Bell and R. Jackiw, A PCAC puzzle: $\pi 0 \rightarrow \gamma \gamma$ in the $\sigma$ model, Nuovo Cimento A 60, 47 (1969).

[22] H. B. Nielsen and M. Ninomiya, The Adler-Bell-Jackiw anomaly and Weyl fermions in a crystal, Phys. Lett. B 130, 389 (1983).

[23] A. Westström and T. Ojanen, Designer Curved-Space Geometry for Relativistic Fermions in Weyl Metamaterials, Phys. Rev. X 7, 041026 (2017).

[24] C.-X. Liu, P. Ye, and X.-L. Qi, Chiral gauge field and axial anomaly in a Weyl semimetal, Phys. Rev. B 87, 235306 (2013).

[25] D. T. Son and B. Z. Spivak, Chiral anomaly and classical negative magnetoresistance of Weyl metals, Phys. Rev. B 88, 104412 (2013).

[26] T. L. Hughes, R. G. Leigh, and O. Parrikar, Torsional anomalies, Hall viscosity, and bulk-boundary correspondence in topological states, Phys. Rev. D 88, 025040 (2013).
[27] A. Lucas, R. A. Davison, and S. Sachdev, Hydrodynamic theory of thermoelectric transport and negative magnetoresistance in Weyl semimetals, Proc. Natl. Acad. Sci. USA 113, 9463 (2016).

[28] C.-L. Zhang, S.-Y. Xu, I. Belopolski, Z. Yuan, Z. Lin, B. Tong, G. Bian, N. Alidoust, C.-C. Lee, and S.-M. Huang, Signatures of the Adler-Bell-Jackiw chiral anomaly in a Weyl fermion semimetal, Nat. Commun. 7, 10735 (2016).

[29] H. Sumiyoshi and S. Fujimoto, Torsional Chiral Magnetic Effect in a Weyl Semimetal with a Topological Defect, Phys. Rev. Lett. 116, 166601 (2016).

[30] Z. M. Raines and V. M. Galitski, Enriched axial anomaly in Weyl materials, Phys. Rev. B 96, 161115(R) (2017).

[31] J. Gooth, A. C. Niemann, T. Meng, A. G. Grushin, K Landsteiner, B. Gotsmann, F. Menges, M. Schmidt, C. Shekhar, and V. Süß, Experimental signatures of the mixed axialgravitational anomaly in the Weyl semimetal $\mathrm{NbP}$, Nature (London) 547, 324 (2017).

[32] Z. V. Khaidukov and M. A. Zubkov, Chiral torsional effect, JETP Lett. 108, 670 (2018).

[33] J. Behrends, S. Roy, M. H. Kolodrubetz, J. H. Bardarson, and A. G. Grushin, Landau levels, Bardeen polynomials, and Fermi arcs in Weyl semimetals: Lattice-based approach to the chiral anomaly, Phys. Rev. B 99, 140201(R) (2019).

[34] Y. Ferreiros, Y. Kedem, E. J. Bergholtz, and J. H. Bardarson, Mixed Axial-Torsional Anomaly in Weyl Semimetals, Phys. Rev. Lett. 122, 056601 (2019).

[35] F. de Juan, M. Sturla, and M. A. H. Vozmediano, SpaceDependent Fermi Velocity in Strained Graphene, Phys. Rev. Lett. 108, 227205 (2012).

[36] F. de Juan, J. L. Mañes, and M. A. H. Vozmediano, Gauge fields from strain in graphene, Phys. Rev. B 87, 165131 (2013)

[37] A. Cortijo, Y. Ferreirós, K. Landsteiner, and M. A. H. Vozmediano, Elastic Gauge Fields in Weyl Semimetals, Phys. Rev. Lett. 115, 177202 (2015).

[38] E. Arias, A. R. Hernández, and C. Lewenkopf, Gauge fields in graphene with nonuniform elastic deformations: A quantum field theory approach, Phys. Rev. B 92, 245110 (2015).

[39] A. G. Grushin, J. W. F. Venderbos, A. Vishwanath, and R. Ilan, Inhomogeneous Weyl and Dirac Semimetals: Transport in Axial Magnetic Fields and Fermi Arc Surface States from PseudoLandau Levels, Phys. Rev. X 6, 041046 (2016).

[40] D. I. Pikulin, A. Chen, and M. Franz, Chiral Anomaly from Strain-Induced Gauge Fields in Dirac and Weyl Semimetals, Phys. Rev. X 6, 041021 (2016).

[41] A. Cortijo and M. A. Zubkov, Emergent gravity in the cubic tight-binding model of Weyl semimetal in the presence of elastic deformations, Ann. Phys. 366, 45 (2016).

[42] R. Winkler, Spin-Orbit Coupling Effects in Two-Dimensional Electron and Hole Systems, Springer Tracts in Modern Physics (Springer, Berlin, 2003).

[43] J. Yepez, Einstein's vierbein field theory of curved space, arXiv:1106.2037.

[44] See Supplemental Material at http://link.aps.org/supplemental/ 10.1103/PhysRevResearch.1.032006 for details of technical derivations and additional information.

[45] G. E. Volovik, Black hole and Hawking radiation by type-II Weyl fermions, JETP Lett. 104, 645 (2016). 
[46] G. E. Volovik and K. Zhang, Lifshitz transitions, Type-II Dirac and Weyl fermions, event horizon and all that, J. Low Temp. Phys. 189, 276 (2017).

[47] H. Liu, J.-T. Sun, H. Huang, F. Liu, and S. Meng, Fermionic analog of black hole radiation with a super high Hawking temperature, arXiv:1809.00479.

[48] S. H. Shenker and D. Stanford, Black holes and the butterfly effect, J. High Energy Phys. 03 (2014) 067.

[49] A. Kitaev, A simple model of quantum holography, talks at KITP, 2015, http://online.kitp.ucsb.edu/online/entangled15/ kitaev.

[50] J. Maldacena, S. H. Shenker, and D. Stanford, A bound on chaos, J. High Energy Phys. 08 (2016) 106.

[51] Y.-G. Chen, X. Luo, F.-Y. Li, B. Chen, and Y. Yu, Quantum chaos associated with emergent event horizon in transition layer between type-I and type-II Weyl semimetals, arXiv:1903.10886 [cond-mat.mes-hall].

[52] W. G. Unruh, Experimental Black-Hole Evaporation? Phys. Rev. Lett. 46, 1351 (1981).

[53] W. G. Unruh, Sonic analogue of black holes and the effects of high frequencies on black hole evaporation, Phys. Rev. D 51, 2827 (1995).

[54] H. Shapourian, T. L. Hughes, and S. Ryu, Viscoelastic response of topological tight-binding models in two and three dimensions, Phys. Rev. B 92, 165131 (2015).

[55] M. A. H. Vozmediano, M. I. Katsnelson, and F. Guinea, Gauge fields in graphene, Phys. Rep. 496, 109 (2010).

[56] J. M. Luttinger, Theory of thermal transport coefficients, Phys. Rev. 135, A1505 (1964). 